\title{
By providing hospice/palliative care education to registered nurses does it improve their comfort level when communicating end of life choices, including hospice with patients and families?
}

\begin{abstract}
Hospice is defined as "a special concept of care designed to provide comfort and support to patients and their families when a life limiting illness no longer responds to cure-oriented treatments". ${ }^{1}$ The benefits of using hospice includes proper medication administration for pain and other controllable symptoms', decreases cost, increases patient/family satisfaction and a decrease in caregiver stress throughout the stay. Why the delay in a patients' referral to hospice care?
\end{abstract}

Objectives: The purpose of this study is to identify if education about hospice/palliative care services improve the nurses' comfort level on communicating to patient regarding end of life issues and hospice.

Design: Pretest-posttest design.

Subjects: Oncology nurses at Seattle based oncology site.

Outcomes Measured: Reflection of prior education on hospice, palliative and hospice care awareness, ability to refer patient to hospice, comfort of talking about hospice, and confidence in knowing when to talk about hospice.

Results: Twenty-eight participants, the average year of nursing was 7.2 years. The nurses had the highest increase comparing pre and post survey in the category of how to initiate hospice services for the patient. Every category measured show an increase in post scores compared to pre-scores. The largest increase was 2.5 points when asked on how comfortable the nurses were in initiating hospice services.

Conclusion: Education of hospice and end of life concepts can prepare healthcare providers to engage in conversations and better manage the difficult conversation about death. Palliative and hospice care education initiatives are lacking in the oncology field and, if provided, nurses would take advantage of the opportunity.

Keywords: selective transfer, superficial layer, structural analysis, intensity x-rays, width of diffraction lines, crystalline network constant
Volume 2 Issue $6-2018$

\author{
Cassandra Hayes RN, San So RN, Abigail \\ Mitchell RN \\ D'Youville College, New York, USA
}

Correspondence: Abigail Mitchell RN, D’Youville College, Buffalo, New York, USA, Tel 7I6-829-82।8,

Email mitchela@dyc.edu

Received: October 29, 2018 | Published: December 28, 2018

\section{Problem statement}

According to the Centers of Disease Control (CDC), there will be over 900,000 to one million new cases of cancer by the year 2020 . The number of deaths related to cancer is also expected to increase by eight to fifteen percent. Many of those patients may be referred to hospice, when the patient is expected to have six months or less to live. Hospice is a special type of care in which medical, psychological, and spiritual support are provided to patients and their loved ones when cancer therapies are no longer controlling the disease. Hospice focuses on controlling pain and other symptoms of illness, so patients can remain as comfortable as possible near the end of life. To be considered for hospice, the patient will need a referral from their doctor and have a less than six months to live. Many times, hospice referrals are a result from end-of-life (EOL) discussions. EOL discussions offer patients the opportunity to define their goals and expectations for the medical care that they want to receive near death. But, these discussions also mean confronting the limitations of medical treatments and the reality that life is finite, both of which may cause psychological distress. ${ }^{2}$
Many providers do not have conversations with patients and their families regarding hospice until it is too late. There are many studies that show members of the patient's care team, oncology nurses for example, do not feel adequately prepared to engage in lengthy, honest, and emotionally draining discussions about end of life care. How can oncology nurses increase their comfort levels when talking to patients about hospice? What are the barriers for the oncology nurse preventing these conversations from happening at the right time? Does a nurse need to be an expert about hospice to feel comfortable having these conversations?

\section{Review of literature}

The literature review was conducted using the databases of CINAHL, PsychINFO, PubMed, Cochrane Library of Systematic Reviews and Medline from 2000 to 2018. Search terms used: communication, hospice, end-of-life barriers, oncology nursing perspective, hospice AND Watson's Theory, hospice AND referrals, and palliative care AND cost, oncology nursing AND hospice. Twelve 
articles were used in this review after applying the exclusion criteria of articles not relating to this question.

This study is going to determine if education provided to oncology nurses will increase their comfort levels when talking to patients about hospice. One of the main questions to be addressed is how comfortable oncology nurses feel having the end of life discussion with their patients. Nevidjon \& Mayer "Death Is Not an Option" that focused on this particular question. The study speaks of asking patients how they want to live their lives during their last weeks. This is a very important question as the answer will reflect when the appropriate time would be to refer the patient to hospice.

Communicating about the end-of-life with patients has been reported as one of the most difficult and stressful conversations for patients and nurses. Barriers of effective communication and referring to hospice were found from both medical staff and patients. Many nurses and medical professionals reported multiple barriers in effective communication to patients and their families regarding end of life care or hospice. ${ }^{4}$ Barriers for nurses presented in several of the studies included the lack of education regarding hospice services, the nurses feeling uncomfortable when talking about end of life care, the staff is unsure of the plan of care and the nurses are afraid to portray themselves of giving up hope to the family and patient. ${ }^{5}$ The patient barriers mainly focused on wanting to keep fighting, afraid of letting down family and care team, family unable to care for patient at home and patient does not know what hospice could provide for them. ${ }^{6}$

Nevidjon \& Mayer ${ }^{3}$ also talked about how cost, fear of abandonment and differences in patient and family wishes cause concerns. These barriers are all things that need to be addressed when speaking with patients and families regarding end of life care. Nevidjon \& Mayer $^{3}$ suggest that oncology nurses should make sure their own health care decisions are made, talk about their own decisions with their family and assist their own families with health care decisions as a way of practicing to overcome the barriers.

Nurses were deemed the most appropriate role to talk to patient and their families about transition to hospice. Gulay \& Gulistan did a study that showed the nurse-patient relationship was viewed by patients and families as the most memorable aspect of treatment. Nurses are in touch with patient and families more often than anyone on the care team. The patients seem to trust the nurses and need guidance in many instances while making decisions. Patient and families often communicate about symptoms, but rarely initiate conversations about end of life. The study by Boyd et al. ${ }^{4}$ reported many of the conversations regarding advanced planning, end of life plan and hospice was initiated by nurses. Some patients did initiate the hospice conversation, but comparatively low compared to medical staff initiation.

Communication is key to the nurses and the patient. Effective communication was another barrier and mainly staff felt not properly educated to manage their emotions regarding patients dying, providing proper information regarding hospice and being honest regarding patient's current health status. ${ }^{8}$ Oncology nurses voiced in a study done in Canada, that the need to develop effective communication is pivotal in providing psychosocial care for adult patients with cancer. Without clear communication on where the aggressive treatment will lead the oncology patient and wondering if the patient is aware of the potential effects on quality of life, many oncology nurses felt anger and emotional distress. ${ }^{9}$ Julie Ann Cronin \& Susan Finn ${ }^{10}$ did a study looking at appropriate education for nurses on communication and what affects did have on the nurses. The study used a detailed
COMFORT curriculum. COMFORT stands for communication, orientation and opportunity, mindful presence, family, openings, relating, and team. Twenty nurses participated in the study. Each participant completed a twenty-six item pre and post surveys that assess positive and negative attitudes toward learning communication skills. Nurses received an intervention; a four-hour education session on using COMFORT communication skills and then completed the post survey. The pre and post scores were not statically significant, but the feedback from many of the nurses were positive. The nurses felt the education provided them with tools for better communication with patient in the oncology setting. ${ }^{10}$

It is important to know the facts about hospice when speaking with patients. One topic to talk to patient and families about is the potential financial burden. Financial burden for the patient and families starts with the very first intervention and may not end when the patient passes away. Schub ${ }^{11}$ wrote an evidenced based care sheet regarding healthcare costs of hospice. According to Schub" "In the U.S. the Medicare Hospice Benefit covers the cost of HC for Medicare beneficiaries". Terminally ill patients and families have hospice as an option through private and government funding programs; however, hospice services are not always used to the fully extend or even at all. ${ }^{4}$ One study done in the United Kingdom, showed many families felt there were too many unnecessary interventions done when they understood death was imminent. The additional testing and workups during the last days of life for their loved ones seemed wasteful when the researchers interviewed bereaved families. The study also pointed out the additional testing caused distress to the patient during the test. The families felt guilty after the death of the family member because they allowed the medical team to carry through the unnecessary testing. The study later discusses that kind of distress calls for better education for the medical staff.$^{12}$ The oncology nurse can provide this small amount of information to the patient and family, then one barrier for the family is gone. The more competent the oncology nurse is regarding hospice information, the more comfortable the nurse will feel when having this discussion.

When the oncology nurses determine that they do not feel adequately prepared to talk about end of life care, then we must figure out how to better educate them. Churma ${ }^{13}$ wrote an article discussing why nurses feel they lack education when talking about and providing end of life care titled "Dedicated Education Unit Model in a Hospice and Palliative Care Setting". The author talks about the different techniques used by staff to educate each other regarding end of life discussions and care. Churma ${ }^{13}$ feels that the roles and responsibilities need to be addressed so that the individuals in these specific roles feel more confident.

The specific roles addressed included adding a preceptor in the facility that was a novice and could educate the staff. It is also important to have an interdisciplinary approach when speaking about patients that could possibly be referred to hospice. This will strengthen the nurses' esteem and provide the ability to answer the underlying questions that may be difficult. Therapeutic communication was also reviewed in this study. It was noted that one student (nurse) found "You learn to listen and use therapeutic communication especially with [patients with] dementia and problems that affect quality of life". ${ }^{13}$ Therapeutic communication will help oncology nurses to listen and communicate with patients and families during this challenging time.

Preparing for end of life discussion should start when nurses are attending colleges/universities; school of nursing. They are 
educated on the foundations except in most cases the most basic; interventions for caring for a patient that is terminally ill. The key being communication within the healthcare system, patient, family, and especially the nurse(s). It is easy to see why nurses, specifically oncology nurses, have difficulty with talking about end of life care. There is minimal education for them and they are not exposed to the questions that the patients and families might seek out. It is our goal to determine if hospice referral education will increase oncology nurse comfort levels when talking to patients about hospice.

\section{Methodology}

The quantitative study used pretest and posttest design. The study was conducted over three to four weeks starting on July 2018 Due to the nature of this study, approval from IRB was not needed. The study was approved by the onsite nurse manager and nurse educator. The study took place at a cancer institute in the Seattle area. Oncology infusion room nurses were provided surveys prior to education session. Each education session lasted for two hours and was conducted by one researcher. The two-hour educational session included educational material about hospice, hospice services, end of life topics and communication styles regarding end of life treatment. A post education survey was given to participants to assess if there were changes in general comfort regarding hospice and communication about hospice to patients. The students had one week to complete the surveys. The data from each pre-survey and post survey was evaluated by both researchers.

Pre and post surveys contain the same statements for the participants to rate. The scale was a one to five Likert scale. Rating of one means strongly disagrees, two means disagree, three means neutral, four means agree and five means strongly agrees. The participants were asked to rate the followings statements on both surveys:

a. I have been properly educated to speak with patient and families regarding end of life care,

b. I feel comfortable when speaking to patient and families regarding end of life,

c. I can provide care as patient and families desire end of life care,

d. I can identify the problem the patient is having and be able to speak with a physician, suggesting end of life care at the appropriate time, e. I understand hospice philosophies and services,

f. I know how to initiate the process to help a patient and family start hospice services, if a patient or family member question me about hospice,

g. I feel confident that I can explain it to them and prior education/ experience has prepared me to talk about hospice and end of life goals with my patient.

We also had a few demographic questions, one question asked the nurses how long they have been an RN, this question is important when we think about Novice to Expert Theory and Patricia Benner.

\section{Results}

Of the twenty-eight participants the average year of nursing was 7.2 years. When rating the statements;

a. I was properly educated to speak with patient and families regarding end of life care, about two of the twenty-eight agreed with that statement on the pretest survey. A majority of the nurses strongly disagreed with the statement and three were neutral for the rating. The same statement showed the lowest average score compared to the other questions. The average pre-survey score was 1.64 .

b. The next lowest scoring pre-test statement was, I feel comfortable when speaking to patient and families regarding end of life, with the average pre-survey rating being 1.82 .

c. Most nurses felt comfortable with caring for patient during end of life, with an average pre-survey score of 2.57. The average increase in post survey rating was about 0.99 over all eight measured outcomes.

d. The highest increase comparing pre and post survey in the category of how to initiate hospice services for the patient, was 2.5 points. This increase in pre and post scores provides evidence that education is needed.

e. The post survey score for the statement, if a patient or family member question me about hospice, I feel confident that I can explain it to them, had the second highest increase. The difference was 1.97 from pre and post scores, which supports the education is needed to better prepare nurses and increase their comfort in end of life (Table 1).

Table I Paired samples statistics

\begin{tabular}{|c|c|c|c|c|c|}
\hline & & Mean & $\mathbf{N}$ & Std. deviation & Std. error mean \\
\hline \multirow[t]{2}{*}{ Pair I } & $\begin{array}{l}\text { I have been properly educated to speak with patient and families regarding end of life care } \\
\text { PRE }\end{array}$ & 1.64 & 28 & 0.951 & 0.18 \\
\hline & $\begin{array}{l}\text { I have been properly educated to speak with patient and families regarding end of life care } \\
\text { POST }\end{array}$ & 3.21 & 28 & 1.343 & 0.254 \\
\hline \multirow[t]{2}{*}{ Pair 2} & I feel comfortable when speaking to patient and families regarding end of life PRE & 1.82 & 28 & 1.02 & 0.193 \\
\hline & I feel comfortable when speaking to patient and families regarding end of life POST & 3.14 & 28 & 1.113 & 0.21 \\
\hline \multirow[t]{2}{*}{ Pair 3} & I can provide care as patient and families desire end of life care PRE & 2.57 & 28 & 1.103 & 0.208 \\
\hline & I can provide care as patient and families desire end of life care POST & 3.71 & 28 & 0.897 & 0.169 \\
\hline \multirow[t]{2}{*}{ Pair 4} & $\begin{array}{l}\text { I can identify the problem the patient is having and be able to speak with a physician, } \\
\text { suggesting end of life care at the appropriate time PRE }\end{array}$ & 2.68 & 28 & 1.156 & 0.219 \\
\hline & $\begin{array}{l}\text { I can identify the problem the patient is having and be able to speak with a physician, } \\
\text { suggesting end of life care at the appropriate time POST }\end{array}$ & 3.39 & 28 & 1.066 & 0.201 \\
\hline
\end{tabular}


Table Continued...

\begin{tabular}{|c|c|c|c|c|c|}
\hline & & Mean & $\mathbf{N}$ & Std. deviation & Std. error mean \\
\hline \multirow[t]{2}{*}{ Pair 5} & I understand hospice philosophies and services PRE & 2.43 & 28 & 1.034 & 0.195 \\
\hline & I understand hospice philosophies and services POST & 3.79 & 28 & 0.917 & 0.173 \\
\hline \multirow[t]{2}{*}{ Pair 6} & I know how to initiate the process to help a patient and family start hospice services PRE & 2.18 & 28 & 1.278 & 0.242 \\
\hline & $\begin{array}{l}\text { I know how to initiate the process to help a patient and family start hospice services } \\
\text { POST }\end{array}$ & 4.68 & 28 & 0.548 & 0.104 \\
\hline \multirow[t]{2}{*}{ Pair 7} & $\begin{array}{l}\text { If a patient or family member question me about hospice, I feel confident that I can } \\
\text { explain it to them PRE }\end{array}$ & 2.21 & 28 & I.197 & 0.226 \\
\hline & $\begin{array}{l}\text { If a patient or family member question me about hospice, I feel confident that I can } \\
\text { explain it to them POST }\end{array}$ & 4.18 & 28 & 0.67 & 0.127 \\
\hline \multirow[t]{2}{*}{ Pair 8} & $\begin{array}{l}\text { Prior education/experience has prepared me to talk about hospice and end of life goals } \\
\text { with my patient PRE }\end{array}$ & 2.18 & 28 & 1.124 & 0.212 \\
\hline & $\begin{array}{l}\text { Prior education/experience has prepared me to talk about hospice and end of life goals } \\
\text { with my patient POST }\end{array}$ & 2.93 & 28 & 0.858 & 0.162 \\
\hline
\end{tabular}

\section{Limitations}

One limitation of this study was the limited variety of staff or type of facility. The study was only done at an outpatient oncology facility. For future studies, it could include a combination of outpatient and inpatient setting. The type of nursing mainly in this study was oncology, but with future studies it would be beneficial to include more intensive care nursing. The number of participants was also small in this study and the scale of the Likert scale was small, which affected statistical significance of the data. For future, studies a larger population and a larger scale would produce more meaningful data. The education sessions were two hours, which limited the amount of information the research was able to convey to the participants. A longer education session would allow additional information to be added and may influence the post survey outcomes. ${ }^{14-16}$

\section{Conclusion}

Talking about end of life and hospice options is a discussion nurses and other health care providers should have with their patients. One hundred percent of the population will come to the time where this is the most important conversation. A way to make sure these important, emotionally charged end-of-life care talks happen may be to make them routine and part of the treatment plan. The data shows that all 28 nurses felt more comfortable talking about end-of-life care after the education was give. Education of hospice and end-of-life concepts can prepare healthcare providers to engage in conversations and better manage the difficult conversation about death. Palliative and hospice care education initiatives are lacking in the oncology field and, if provided, healthcare providers would take advantage of the opportunity.

\section{Acknowledgments}

None.

\section{Conflicts of interest}

The authors declare there is no conflict of interest.

\section{References}

1. Iversen A, Sessanna L. Utilizing Watson's theory of human caring and Hills and Watson's emancipatory pedagogy to educate hospital-based multidisciplinary healthcare providers about hospice. International Journal for Human Caring. 2012;16(4):42-49.
2. Casarett DJ, Quill TE. "I'm Not Ready for Hospice”: Strategies for timely and effective hospice discussions. Ann Intern Med. 2007;146(6):443449.

3. Nevidjon BM, Mayer DK. Death is not an option, how you die is - Reflections from a career in oncology nursing. Nurs Econ. 2012;30(3):148-152.

4. Boyd D, Merkh K, Rutledge DN, et al. Nurses' perceptions and experiences with end-of-life communication and care. Oncol Nurs Forum. 2011;38(3):E229-E239.

5. Weiner JS, Cole SA. Three principles to improve clinician communication for advance care planning: overcoming emotional, cognitive, and skill barriers. J Palliat Med. 2004;7(6):817-829.

6. Bhatt A, Mitchell A. Effective collaborative communication in hospice care. Palliative Medicine \& Care: Open Access. 2015;2(1):1-13.

7. Gulay IC, Gulistan Y. The relationship between cancer patients' perception of nursing care and nursing attitudes towards nursing profession. Asia Pac J Oncol Nurs. 2014;1(1):16-21.

8. Schulman-Green D, McCorkle R, Cherlin E, et al. Nurses' communication of prognosis and implications for hospice referral: A study of nurses caring for terminally ill hospitalized patients. Am J Crit Care. 2005;14(1):64-70

9. Watts R, Botti M, Hunter M. Nurses' perspectives on the care provided to cancer patients. Cancer Nursing. 2010;33(2):E1-E8.

10. Cronin JA, Finn S. Implementing and evaluating the COMFORT communication in palliative care curriculum for oncology nurses. Journal of Hospice \& Palliative Nursing. 2017;19(2):140-146.

11. Schub TB. Hospice care: Health care costs. CINAHL Nursing Guide. Ipswich, MA, USA: Nursing Reference Center Plus; 2016.

12. Small N, Barnes S, Gott M, et al. Dying, death and bereavement" a qualitative study of views of carers of people with heart failure in the UK. BMC Palliat Care. 2009;8(6):1-10.

13. Chmura JQ. Dedicated education unit model in a hospice and palliative care setting. Nurse Educ. 2016;41(1):25-30.

14. Davis A, Maisano P. Patricia Benner: Novice to expert-A concept whose time has come (Again). Oklahoma Nurse. 2016;61(3):13-15.

15. Fischer S, Egan K, Kassner C, et al. Healthcare professional education: A unique role for hospices. Am J Hosp Palliat Care. 2005;22(1):32-40.

16. Hartjes TM. Improving care at the end of life: Creating hospice in place. Crit Care Nurse. 2017;37(5):93-96. 